\title{
Potential Application of Color and Hyperspectral Images for Estimation of Weight and Ripeness of Oil Palm (Elaeis guineensis Jacq. var. tenera)
}

\author{
Phorntipha Junkwon*1), Tomohiro Takigawa ${ }^{1)}$, Hiroshi Okamoto ${ }^{2)}$, Hideo Hasegawa ${ }^{1)}$, \\ Masayuki Koike ${ }^{1)}$, Kenshi Sakai ${ }^{3)}$, Jindawan Siruntawineti ${ }^{4}$, Win Chaeychomsrii), \\ Nuttha Sanevas ${ }^{5)}$, Palat Tittinuchanon ${ }^{6}$ and Banshaw Bahalayodhin ${ }^{7)}$
}

1) Graduate School of Life and Environmental Sciences, University of Tsukuba, 1-1-1 Tennodai, Tsukuba, Ibaraki, 305-8572, Japan

2) Research Faculty of Agriculture, Hokkaido University, Kita-9, Nishi-9, Kita-ku, Sapporo 060-8589, Japan

3) Faculty of Agriculture, Tokyo University of Agriculture and Technology, 3-5-8, Saiwai, Fuchu, Tokyo, 183-8509, Japan

4) Department of Zoology, Faculty of Science, Kasetsart University, Bangkok, 10900, Thailand

5) Department of Botany, Faculty of Science, Kasetsart University, Bangkok, 10900, Thailand

6) Oil Palm Research Centre, Univanich Palm Oil Public Company Ltd., Krabi, 81110, Thailand

7) Former Associate Professor, Faculty of Engineering at Kamphaengsean, Kasetsart University Kamphasean Campus, Nakhon Pathom, 73140, Thailand

\begin{abstract}
The intent of this study was to develop a technique for weight and ripeness estimation of oil palm (Elaeis guieensis Jacq. var. tenera) bunches from hyperspectral and RGB color images. In the experiments, color and hyperspectral images of the bunch were acquired from four different angles, each differing by 90 degrees. Acquired RGB color images were converted to HSI and $\mathrm{L}^{*} \mathrm{a} * \mathrm{~b}$ color space. Gray-scale thresholds were used to identify the area of the bunch and the area of space between the fruits. The total number of pixels in the bunch and the space were counted, respectively. In the hyperspectral images, the total number of pixels in the bunch was also counted from an image composed of three wavelengths $(560 \mathrm{~nm}$, $680 \mathrm{~nm}$, and $740 \mathrm{~nm}$ ), while the total number of pixels of space between fruits was obtained at a wavelength of $910 \mathrm{~nm}$. From these sets of data, weight-estimation equations were determined by linear regression (LR) or multiple linear regression (MLR). As a result, the coefficient of determination $\left(\mathrm{R}^{2}\right)$ of actual weight and estimated weight were at a level of 0.989 and 0.992 for color and hyperspectral images, respectively. Estimation of oil palm bunch ripeness was also tested. Bunches belonging to 4 classes of ripeness (overripe, ripe, underripe, and unripe) were used for this study. Since ripeness estimation from overall data from a bunch was quite difficult, we focused on the difference in colors or reflectivity of the portion concealed and not-concealed with fronds. Euclidean distances between the test sample and the standard 4 classes of ripeness were calculated, and the test sample was classified into the ripeness class that had the shortest distance from the sample. In the classification based on color image, average RGB values of concealed and not-concealed areas were used, while in hyperspectral images the average intensity values of fruits pixels from the concealed area were used. The results of validation experiments with the developed estimation methods indicated acceptable estimation accuracy, and a possibility for practical use to estimate the ripeness of oil palm bunches.
\end{abstract}

\section{Key words}

oil palm, biodiesel, color image, hyperspectral image, ripeness, Thailand

\footnotetext{
* Corresponding Author

E-mail: pjunkwon@hotmail.com
} 


\section{Introduction}

A worldwide rise in oil prices has made alternative energy sources to be a general public interest. Biodiesel has become a common alternative fuel to run machinery and vehicles (Demirbas 2007). Many oil crops have been studied with regard to their potential for producing biodiesel, including jatropha, sunflowers, soybeans and oil palms (Kalam and Masjuki 2002, Leelawath 2006, Usta et al. 2004, Sarin et al. 2007, Murphy 2007). In Thailand, oil palm plantations have been encouraged to expand so as to increase crude palm oil (CPO) production for a variety of domestic uses. Oil palm (Elaeis guineensis Jacq. var. tenera) is mainly planted in southern Thailand because it is an area of sufficient rainfall and suitable climate compared to other regions. Regarding oil palm production in Thailand, the current data show that 7.6 million tons of fresh fruit bunches (FFBs) were produced in 2007 (FAO 2007). Due to a continuous increase in demand for biodiesel, it is estimated that domestic consumption of biodiesel in Thailand will grow to 31.3 billion liters per year in 2011. This is equivalent to 17.7 million tons of FFBs and it might require 1.46 million hectares of plantation area. To satisfy future demands for oil palm, a project to expand oil palm plantations was approved by the Thai Cabinet and introduced to northern and northeastern of Thailand. However, the yield of oil palm may be low due to insufficient rainfall and an unsuitable climate. Therefore, the expanding plantation area is not the only approach to increasing the CPO. At present, the oil extraction rate in Thailand is $16-17 \%$, while it is $20-24 \%$ in neighboring countries (Leelawath 2006). This low oil extraction rate stems partly from the fact that the decision regarding when to harvest the FFBs is made based only on the purchasing price being paid by the factories rather than on the ideal harvest time. Consequently, unripe or overripe bunches are harvested and sold to the factories without ripeness control.

Free fatty acids are important to oil quality. The recommended free fatty acid level is lower than $5 \%$. Although the free fatty acid content will increase linearly with the presence of overripe bunches, each additional percentage point of unripe bunches can reduce the oil extraction rate by $0.13 \%$ (Siregar 1976 cited by Abdullah et al. 2002). The lack of ripeness control is therefore a major cause of low oil extraction rate and oil quality.

Moreover, FFBs are often intentionally mixed with sand and stones by middlemen to increase the weight of bunches before selling to factories. This illegal act decreases the oil extraction rate and oil quality. Randomized sorting of FFBs before purchasing has been carried out to prevent contamination, but this process is time-consume and labor-intensive. In addition, skilled specialists are not available enough for checking derived FFBs. To solve such problems, a technique for estimating the weight and ripeness of FFBs must be developed.
Fruit color is one of the most significant inspection criteria for fruit identification and is indicative of maturity. Image processing can be used to acquire data regarding the color and size of objects from images captured with a camera. Images can be used to provide data as an analytical tool for food quality and safety control (Gowen et al. 2007). Nondestructive techniques, especially image processing techniques, have been used to study the quality of agricultural products by many researchers, investigating issues such as contamination and defects in apples (Patrick et al. 2004), contamination of poultry (Park et al. 2007), qualities such as ripeness, moisture content, total soluble solids, and $\mathrm{pH}$ of strawberries (Gamal et al. 2007), etc. For oil palm, studies regarding bunch ripeness classifications have been carried out based on such information as RGB color images of fruits or bunches (Ismail et al. 2000, Abdullah et al. 2002, Abdullah et al. 2004).

Recently, hyperspectral images have been used intensively to assess the quality of agricultural products because they can provide more detailed reflectance data than RGB color images. Reflectance of product in different levels of quality can be displayed in distinctive patterns at specific wavelengths. As such, the adoption of hyperspectral imaging techniques has become more feasible for better evaluation of quality parameters.

The objectives of this study were to evaluate the potential applicability of hyperspectral image analysis to estimate the weight and ripeness of FFBs, in comparison with analysis based on RGB color images.

\section{Materials and Methodology}

\section{FFB sampling and preparation}

Standards established by the Department of Agriculture in Thailand classify oil palm bunch ripeness into six classes: unripe, underripe, ripe, overripe, rotten, and empty bunch (Table 1). In the present study, oil palm bunch samples that could be classified into four classes of ripeness (unripe, underripe, ripe, and overripe) were prepared. Ripeness class was determined by a human expert using the conventional method of classification in which the number of detached fruits on the ground is counted before

Table 1 Classification of oil palm bunch

\begin{tabular}{ll}
\hline \multicolumn{1}{c}{ Class } & \multicolumn{1}{c}{ Description } \\
\hline Unripe bunch & $\begin{array}{l}\text { Fruits in bunch are hard. Their colors are black and } \\
\text { there is no detached fruit. }\end{array}$ \\
Underripe bunch & $\begin{array}{l}\text { Fruits in bunch have a reddish black color and one to } \\
\text { nine fruits are detached. }\end{array}$ \\
Ripe bunch & $\begin{array}{l}\text { Fruits in bunch have a reddish orange color and 10 } \\
\text { fruits are detached. }\end{array}$ \\
Overripe bunch & $\begin{array}{l}\text { Fruits in bunch have a reddish orange color and } \\
\text { detached fruits are more than 50 fruits. }\end{array}$ \\
Rotten bunch & $\begin{array}{l}\text { Detached fruits are more than 33\% of total fruits. } \\
\text { Bunch with no fruit. }\end{array}$ \\
\hline
\end{tabular}




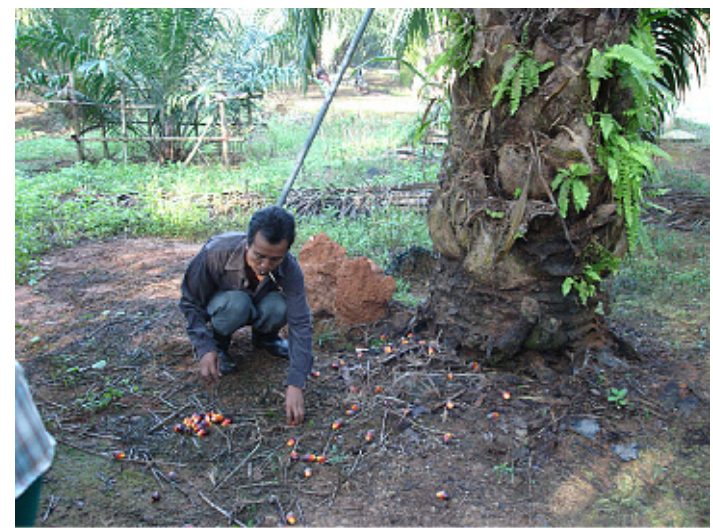

(a) Detached fruits collecting

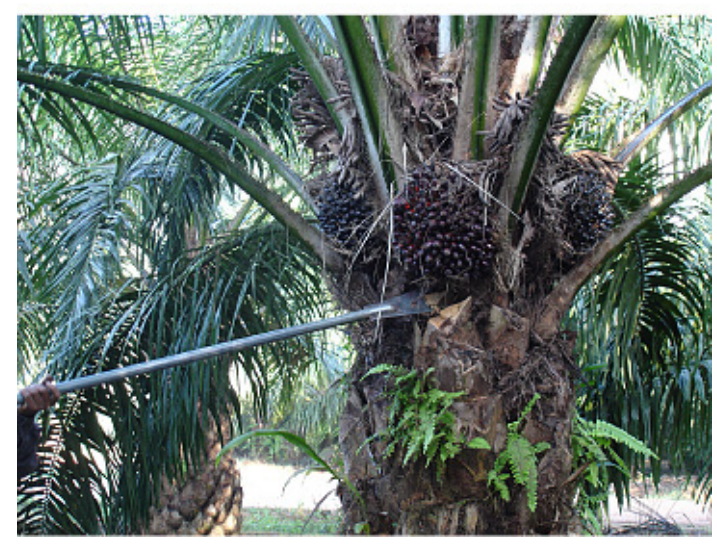

(b) FFBs harvesting

Fig. 1 Oil palm fresh fruits bunch (FFBs) harvesting

harvest. Forty-eight FFBs were harvested in the local plantation in Krabi province, Thailand (Fig. 1). All fiber and dirt were removed with water and a brush to prepare the samples for the experiments, as shown in Fig. 2. These FFBs were dried with a cool air blower before being weighed.

\section{Image acquisition and procession}

A hyperspectral camera (SPECIM, ImSpector V10) and RGB color camera (Sony, Cybershot DSC-P150) were used to acquire images of FFB at four different angles, each differing by 90 degrees. The laboratory hyperspectral imaging system included the following components: (1) an illumination unit consisting of two $150 \mathrm{~W}$ metal-halide lamps with reflectance umbrellas adjusted at $45^{\circ}$ angles, (2) an FFB turn table to rotate the FFB by 90 degrees, (3) a hyperspectral camera with a scanning system, and (4) a white color screen. The hyperspectral camera can acquire spectral images at a wavelength range of 400-1000 nm. Since the hyperspectral camera can scan only line image, the plane image is taken by moving the camera's optical axis with a set of scanning system consists of electrical stepping motor and gear set as shown in Fig. 3. RGB color images of FFB are also captured for comparison with the hyperspectral images.

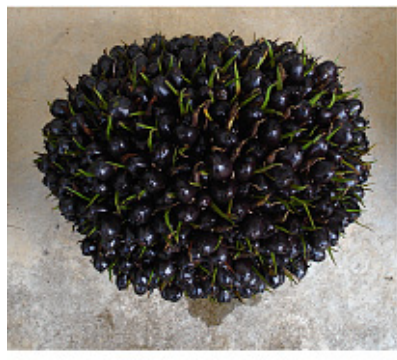

(a) Unripe bunch

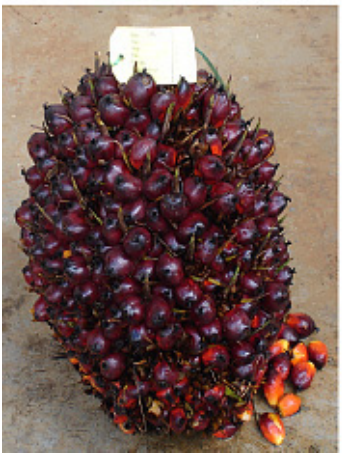

(c) Ripe bunch

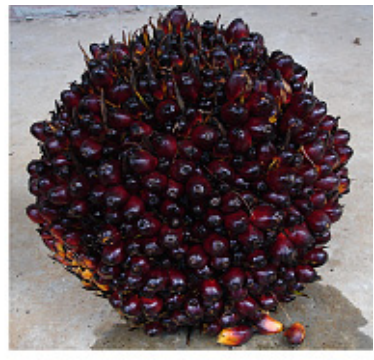

(b) Underripe bunch

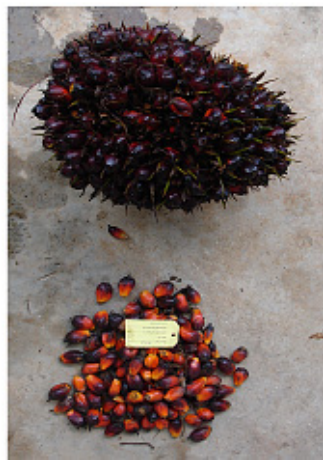

(d) Overripe bunch
Fig. 2 Oil palm bunch image in four ripeness stages

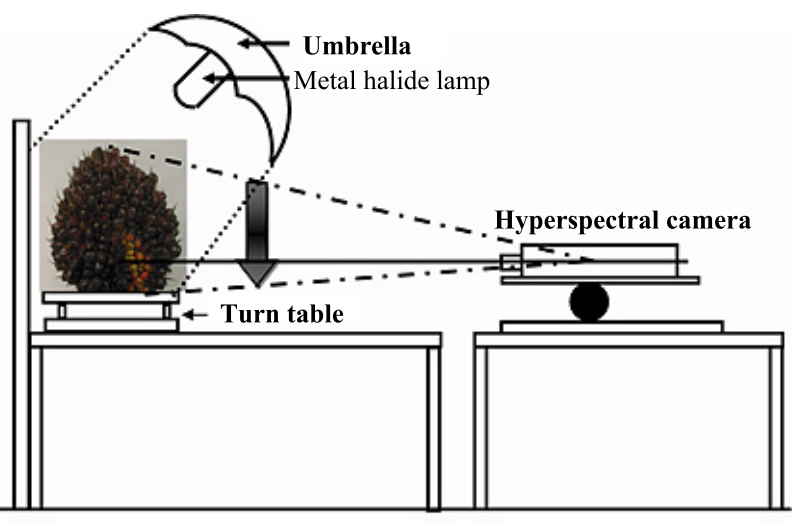

(a) Hyperspectral images capturing method

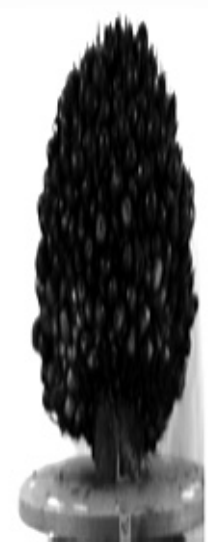

(b) Oil palm image from wavelengths at $750 \mathrm{~nm}$

Fig. 3 Hyperspectral images capturing. 


\section{Weight Estimation}

Forty-eight FFBs with weights in the range of $2-42 \mathrm{~kg}$ were prepared for the experiments. An oil palm bunch consists of clusters of fruits. Oil palm fruit is a sessile drupe borne in a large compact bunch. Fruits develop in size as they increase in maturity, as reported by Thomas et al. (1971). The space inside the bunch may be reduced as the fruits in the bunch become more mature.

\section{Weight estimation from RGB images}

Since digital images are composed of pixels, size features such as area can be acquired by counting the number of pixels in the images. For bunch weight estimation, features from bunch images such as the bunch area or bunch geometry were used as indexes. The bunch geometry, however, was extremely difficult to measure due to shape irregularities; thus the bunch area as shown in the images was used in this study.

In order to improve the image quality, pixel pre-processing must be performed to enhance some image features for specific applications. Color space transformation is one of the most common methods. Among the applications where color space transformation has been used, most are based on HSI (hue, saturation, and intensity) color space. HSI color space is effective for color image distinguishment (Du and Sun 2004). Usually, color images are taken by a digital device and saved in the three-dimensional RGB (red, green, and blue) color space. Images can be extracted into red, green and blue band images. To create hue, saturation and intensity band images in HSI color space, color images of oil palm bunch were transformed from the RGB color space using image processing software (Halcon 7.0, MvTec Germany). With a trial and error approach, gray-scale values ranging from zero to 60 in intensity band image showed the most efficient method of detecting oil palm bunch area from background. The total number of bunch pixels $\left(\mathrm{Ob}_{\mathrm{t}}\right)$ counted from the detected bunch area was used for the weight estimation. However, since space inside the bunch decreases with advancing ripeness due to fruit growth, the classes of ripeness much also be taken into account in the weight estimation. We made an assumption that the total area of space between fruits in bunch images is related to the space inside the oil palm bunch. In the same manner as for the bunch area, the total number of pixels of space between fruits $\left(\mathrm{Os}_{\mathrm{t}}\right)$ was counted. Since the space between fruits cannot be clearly identified from the images of HSI color space, $\mathrm{L}^{*} \mathrm{a} \mathrm{b}^{*}$ color space, which is widely used by many researchers (Abdullah et al. 2001; Abdullah et al. 2002; and Vizhanyo and Felfodi 2000), was selected to detect the area of space between fruits. With a trial and error method, the space between fruits can be extracted effectively by selecting the $\mathrm{L}$ band image in the $\mathrm{L}^{*} \mathrm{a} * \mathrm{~b}$ color space at a grayscale range from zero to 35, as shown in Fig. 4(a). Actually, four images were acquired from 90-degree increments to obtain over- all information for the bunch, and $\mathrm{Ob}_{\mathrm{t}}$ and $\mathrm{Os}_{\mathrm{t}}$ were then calculated by equations (1) and (2).

$$
\begin{aligned}
\left(O b_{t}\right) & =\frac{\sum_{i=0}^{270} O b_{i}}{2}, i=0,90, \ldots, 270 \\
\left(O s_{t}\right) & =\frac{\sum_{i=0}^{270} O s_{i}}{2}, i=0,90, \ldots, 270
\end{aligned}
$$

where $\mathrm{Ob}_{\mathrm{i}}$ is the total number of pixels of the bunch at the $\mathrm{i}^{\text {th }}$ degree of rotation, $\mathrm{Os}_{\mathrm{i}}$ is the total number of pixels of space between fruits at the $i^{\text {th }}$ degree of rotation, and $i$ is the degree of rotation at which the image is acquired.

Weight-estimation equations were constructed using linear regression (LR) and multiple linear regression (MLR). The weight-estimation equation can be simply written as equations (3) and (4) for linear and multiple linear regression, respectively.

$$
\begin{aligned}
& W=a\left(O b_{t}\right) \\
& W=b\left(O b_{t}\right)+c\left(O s_{t}\right)+d
\end{aligned}
$$

where $\mathrm{Ob}_{\mathrm{t}}$ is the total number of pixels of the bunch, $\mathrm{Os}_{\mathrm{t}}$ is the total number of pixels of space between fruits, $\mathrm{W}$ is the oil palm bunch weight $(\mathrm{kg})$, and $\mathrm{a}, \mathrm{b}, \mathrm{c}, \mathrm{d}$ are constant values.

\section{Weight estimation from hyperspectral images}

Hyperspectral images were obtained with software developed by Okamoto et al. (2006). Images were extracted from wavelengths at $400 \mathrm{~nm}$ to $1000 \mathrm{~nm}$ with an average 10-nm interval, and a total of 60 images were obtained. In addition, an image composed of three wavelengths $(560 \mathrm{~nm}, 680 \mathrm{~nm}$, and $740 \mathrm{~nm}$ ) was also provided. Thereby 61 images per 90 degrees of bunch rotation were obtained. For hyperspectral image analysis, the intensity value has widely been used to detect and classify object as reported by Okamoto et al. 2007 and Al-Mallahi et al. 2008. For estimation of the oil palm bunch weight, hyperspectral images were processed in the same manner. The total number of pixels of the bunch and the total number of pixels of space between fruits were counted. Using a trial and error approach, the bunch area was most clearly extracted from the image composed of three wavelengths when the pixels had intensity values between zero and 37 . In contrast, the image of the wavelength at $910 \mathrm{~nm}$ provided the best recognition of the area of space between the fruit when the pixel intensity values stayed in the range between zero and 2, as shown in Fig. 4(b).

Eight FFBs per class of ripeness were used as a weightestimation dataset (calibration dataset), while the rests were used for validation. The quality of the calibration equations are evaluated from the standard error of calibration (SEC), the standard error of prediction (SEP), and the coefficient of determination 


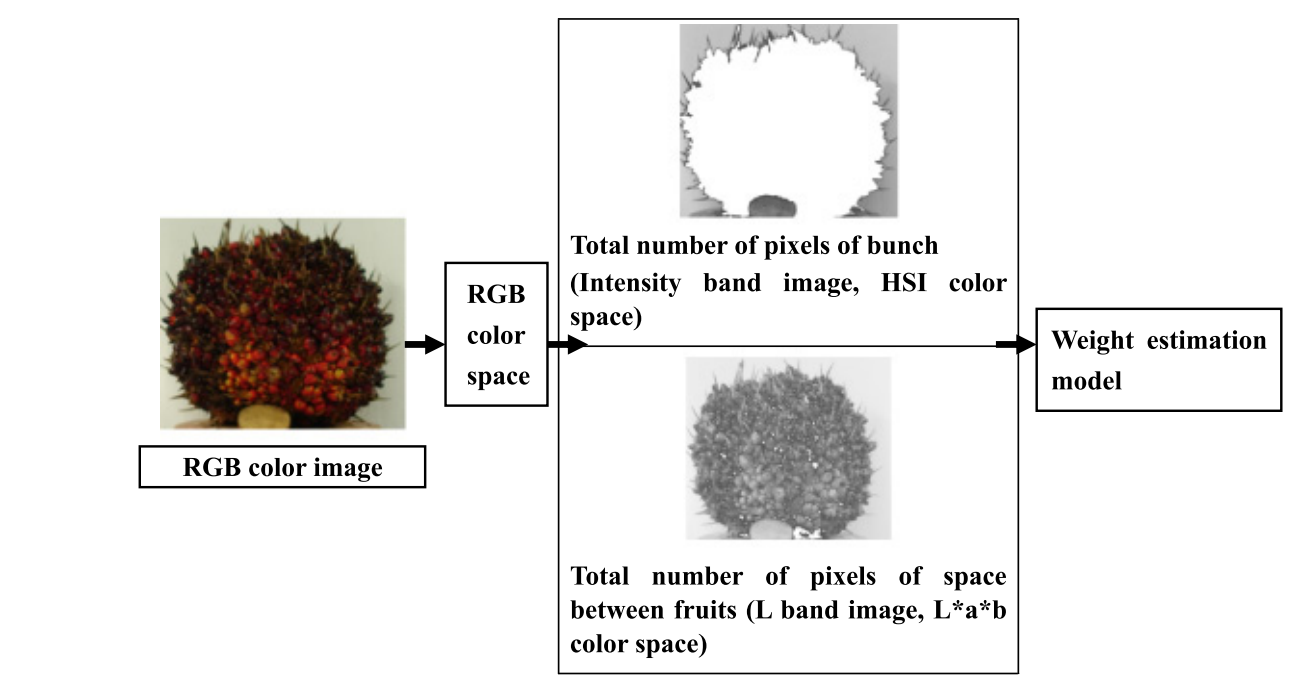

(a) RGB color image

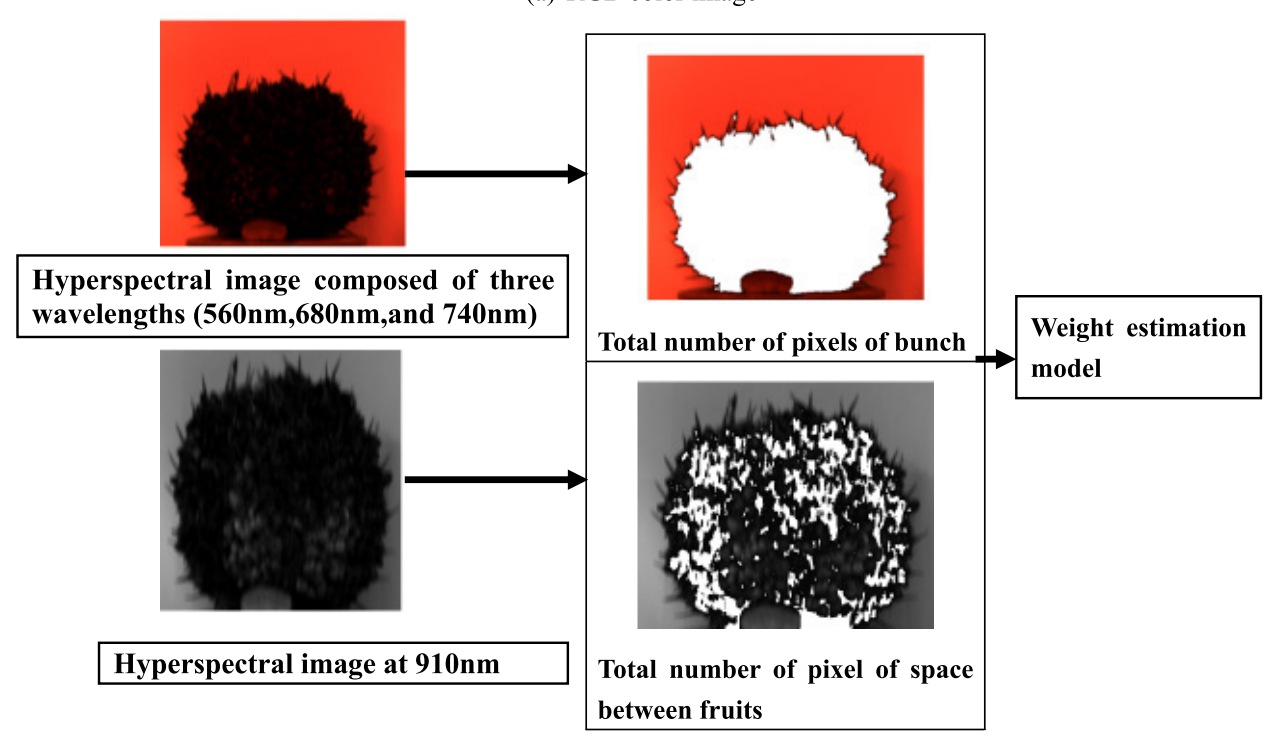

(b) Hyperspectral image

Fig. 4 Concept of FFB weight estimation

$\left(\mathrm{R}^{2}\right)$ between the estimated and actual weight. Lower SEC, lower SEP, higher coefficient of regression, and smaller difference between SEC and SEP mean higher estimation performance. These criteria are defined as follows:

$$
\begin{aligned}
& S E C=\sqrt{\frac{1}{J_{c}-1} \sum_{j=1}^{J_{c}}\left(\hat{y}_{j}-y_{j}\right)^{2}} \\
& S E P=\sqrt{\frac{1}{J_{p}-1} \sum_{j=1}^{J_{p}}\left(\hat{y}_{j}-y_{j}-\text { bias }\right)^{2}} \\
& \text { bias }=\frac{1}{J_{p}} \sum_{j=1}^{J_{p}}\left(\hat{y}_{j}-y_{j}\right)
\end{aligned}
$$

where $\hat{y}_{j}$ is the estimated weight in FFB number $\mathrm{j}, y_{j}$ is the actual weight in FFB number $j, J_{c}$ is the number of FFBs in the calibra- tion dataset, and $\mathrm{J}_{\mathrm{p}}$ is the number of FFBs in the validation dataset.

\section{Ripeness Estimation \\ Ripeness estimation by RGB color images}

Of the 48 bunches, 24 were selected and classified by referring to the standard of Department of Agriculture in Thailand. Four bunches per class of ripeness were used as a calibration dataset, while other two bunches per class were used for validation tests. For estimating the ripeness of oil palm bunch, colors of oil palm fruits can be a good index regarding ripeness, but accurate ripeness classification based on RGB images requires more than 100 samples per class, and the percentage of correct classification was around 90\% (Abdullah et al.2002). However, there are many fruits in one bunch. Therefore, information from pixels of the fruits in different parts of the oil palm bunch image was processed 


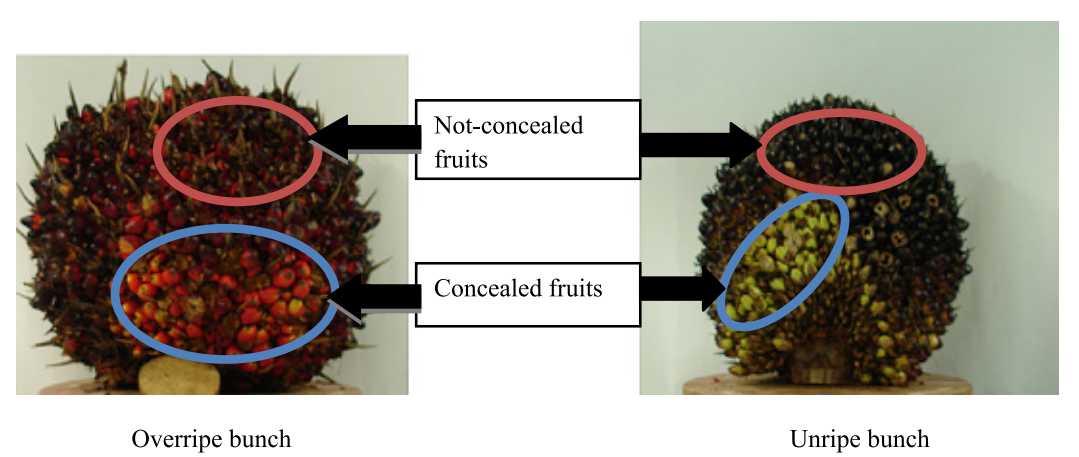

Fig. 5 Concealed and not concealed fruits

for the ripeness estimation to reduce the computational time.

In this research, we focused on the fact that fruits in one bunch have two parts that are different in color: fruits that are concealed by fronds (shade fruits) and those that are not concealed (exposed or normal fruits) as shown in Fig. 5. Four images from different angles with 90 degrees increment were used per bunch. Ripeness classification was attempted based on the difference in color between concealed and not-concealed fruits.

However, selection of the concealed and not-concealed parts is a relatively difficult task; as such, both manual and automatic sampling methods were also tested. To collect sample of pixels, a program to select and save data from selected pixels was developed by image processing software (Halcon.7.0, MvTec Germany) for manual and automatic sampling methods. In the manual sampling method, 10 pixels of shade fruits were selected first by clicking on the concealed part of the first image of bunch, followed by 10 pixels of normal fruits on the not-concealed part of the image. In order to avoid sampling in the same pixel, a coordinate of the selected pixel was recorded while its RGB values were saved in a data file. The same procedure was performed for other images that captured from three different angles of bunch. The average RGB values of fruits from shade fruits (Rs, Gs, Bs) and normal fruits (Rn, Gn, Bn), and then (Rs-Rn, Gs-Gn, and Bs-Bn) and (Rs-Gs, Rs-Bs, Gs-Bs, Rn-Gn, Rn-Bn, and Gn-Bn,) were also computed.

To propose a more practical approach to ripeness estimation, an automatic sampling method was used with the color images. Firstly, a segmentation of bunch area from background was done to make a sampling easier. Gray-scale ranging zero to 60 from intensity band image was used to segment the bunch area from the original color images. The segmented bunch area image shown in Fig. 6 was used for automatic ripeness estimation.

Then, a trial to detect shade and normal fruits was performed by transforming segmented bunch area image to images in HSI color space. The most suitable range of gray-scale values to detect the area of shade and normal fruits was found between range of 77 to 255 and 5 to 20 of saturation band images, respectively.

All pixels from the area of shade and normal fruits were col-

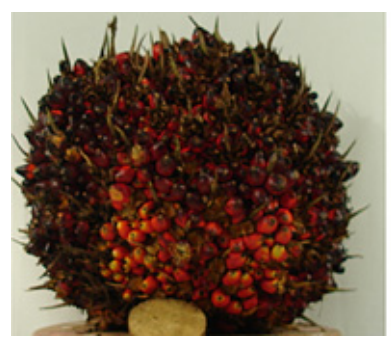

(a) Original image

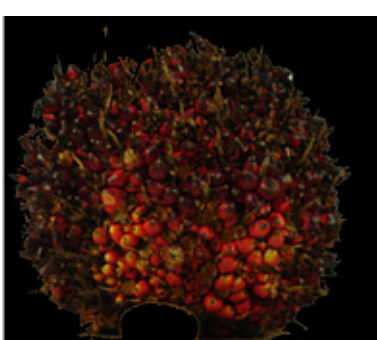

(b) Segmented bunch area image
Fig. 6 Background segmentation of FFB

lected as RGB values and calculated as average RGB values in the same manner as the manual sampling method.

\section{Ripeness estimation by hyperspectral images}

For the hyperspectral images, 4 images per bunch with 90 degree of different angle were employed to obtain sampling pixels. Twenty pixels of shade fruits per image were randomly picked from shade fruits and recorded by software developed by Okamoto et al. (2006). Totally 80 pixels per bunch were collected. Note that each pixel has intensity values from 400 to $1000 \mathrm{~nm}$ with an average 10-nm interval. To select the suitable wavelengths for the ripeness estimation, the significance in differences between average intensity values was tested. The statistical results showed significant differences in ripeness at wavelengths of 730,740 , and $750 \mathrm{~nm}$.

\section{Classification method}

Principal components analysis (PCA) and Euclidean distance were used to identify the groups having different ripenesses. PCA is a method for compressing variables and generating a new set of variables which we refer to as the principle components. The distribution of the components 1 (PC1) and 2 (PC2) can help to clarify variable classification.

Euclidean distance was also useful for classification between classes of ripeness. The Euclidean distance (Dis) between two points, $X\left(x_{1}, x_{2}, \ldots, x_{m}\right)$ and $Y\left(y_{1}, y_{2}, \ldots, y_{m}\right)$, in the $m$-th dimensional space was calculated by Equation 8. First, as the standard characteristic values of ripeness classes, centroid, of the average RGB values and the differences of the average RGB of color 
images or the average intensity values of hyperspectral images were calculated. Next, the Euclidean distance from an unknown sample to the centroid for each class of ripeness was computed. The Euclidean distance between centroid ' $\mathrm{x}$ ' to sample' $y$ ' was obtained by the next equation.

$$
D i s=\sqrt{\left(x_{1}-y_{1}\right)^{2}+\left(x_{2}-y_{2}\right)^{2}+\ldots+\left(x_{m}-y_{m}\right)^{2}}
$$

In the classification process, the distances from a sample to the centroid for each class were calculated, and the sample is classified into the class for which the distance is the shortest.

\section{Results and Discussion}

\section{Weight determination}

Table 2 shows the validation results of weight determination of FFBs by using RGB and hyperspectral image. The results of the weight-estimation equations (Equations 9 and 10) by linear regression (LR) displayed a high accuracy in large bunches (weight more than $15 \mathrm{~kg}$ ), but the error in estimated weight of the small bunches (weight lower than $15 \mathrm{~kg}$ ) was over $45 \%$ of the actual weight. On the other hand, when multiple linear regressions (MLR) in Equations 11 and 12 were used, superior results were obtained even for small bunches. The error varied from between 0 to $18 \%$ of actual weight. $\mathrm{R}^{2}$ of the validation dataset yielded approximately 0.915 (LR) and 0.989 (MLR) in the RGB color image but 0.888 (LR) and 0.992 (MLR) in the hyperspectral images. Weight-estimation equations were as follows:

$$
\begin{aligned}
& W=9.58 \times 10^{-5}\left(O b_{t c}\right) \\
& W=3.4 \times 10^{-5}\left(O b_{t p}\right) \\
& W=-3.784+1.074 \times 10^{-4}\left(O b_{t c}\right)-2.221 \times 10^{-5}\left(O S_{t c}\right) \\
& W=-4.928+4.355 \times 10^{-5}\left(O b_{t p}\right)-3.14 \times 10^{-6}\left(O S_{t p}\right)
\end{aligned}
$$

where $\mathrm{W}$ is the FFB weight, $O b_{t c}$ is the total number of pixels for the bunch in the color image, $O b_{t p}$ is the total number of pixels of the bunch in the hyperspectral image, $O S_{t c}$ is the total number of pixels of space between fruits in the color image, and $O S_{t p}$ is the total number of pixels of space between the fruit portion in the hyperspectral image.

The standard error of calibration (SEC) from calibration data set and the standard error of prediction (SEP) from validation data set of equations from 9 to 12 indicted that the best model for estimation of weight of FFBs was equation 12. The results also suggest that the equation obtained by multiple linear regression from

\begin{tabular}{|c|c|c|c|c|c|c|c|c|c|c|}
\hline \multirow{2}{*}{$\begin{array}{l}\text { Bunch } \\
\text { No. }\end{array}$} & \multirow[b]{2}{*}{ Ripeness } & \multirow{2}{*}{$\begin{array}{l}\text { Weight } \\
\text { (kg) }\end{array}$} & \multicolumn{4}{|c|}{ Predicted weight by color images $(\mathrm{kg})$} & \multicolumn{4}{|c|}{ Predicted weight by hyperspectral images $(\mathrm{kg})$} \\
\hline & & & $\begin{array}{c}\text { LR } \\
\text { (Eq. 9) }\end{array}$ & $\begin{array}{c}\text { Error } \\
(\%)\end{array}$ & $\begin{array}{c}\text { MLR } \\
\text { (Eq. 11) }\end{array}$ & $\begin{array}{c}\text { Error } \\
(\%)\end{array}$ & $\begin{array}{c}\text { LR } \\
\text { (Eq. 10) }\end{array}$ & $\begin{array}{c}\text { Error } \\
(\%)\end{array}$ & $\begin{array}{c}\text { MLR } \\
\text { (Eq.12) }\end{array}$ & $\begin{array}{l}\text { Error } \\
(\%)\end{array}$ \\
\hline 1 & Overripe & $29.7^{*}$ & 27.2 & 8.42 & 26.5 & 10.77 & 26.5 & 10.77 & 29.30 & 1.35 \\
\hline 2 & & $24.3^{*}$ & 22.6 & 6.91 & 21.3 & 12.35 & 21.8 & 10.29 & 23.0 & 5.35 \\
\hline 3 & & 3.4 & 6.2 & 82.24 & 3.1 & 8.82 & 6.5 & 91.18 & 2.8 & 17.65 \\
\hline 4 & & 4.6 & 7.4 & 60.87 & 4.5 & 2.17 & 7.9 & 71.74 & 4.6 & 0.00 \\
\hline 5 & Ripe & $23.8^{*}$ & 24.5 & 3.07 & 23.2 & 2.52 & 23.6 & 0.84 & 24.6 & 3.36 \\
\hline 6 & & $23.0^{*}$ & 22.5 & 2.13 & 21.3 & 7.39 & 22.2 & 3.48 & 23.1 & 0.43 \\
\hline 7 & & 4.5 & 7.3 & 61.56 & 4.0 & 11.11 & 7.5 & 66.67 & 4.0 & 11.11 \\
\hline 8 & & 4.9 & 7.8 & 58.98 & 4.8 & 2.04 & 8.3 & 69.39 & 5.3 & 8.16 \\
\hline 9 & Underripe & $21.3^{*}$ & 20.9 & 1.92 & 19.2 & 9.86 & 19.9 & 6.57 & 20.7 & 2.82 \\
\hline 10 & & $17.0^{*}$ & 18.7 & 9.82 & 17.0 & 0.00 & 18.1 & 6.47 & 18.5 & 8.82 \\
\hline 11 & & 6.1 & 8.9 & 45.74 & 5.9 & 3.28 & 9.4 & 54.10 & 7.1 & 16.39 \\
\hline 12 & & 3.8 & 6.7 & 76.84 & 3.5 & 7.89 & 7.0 & 84.21 & 3.8 & 0.00 \\
\hline 13 & Unripe & $25.1^{*}$ & 23.6 & 6.18 & 21.8 & 13.15 & 22.2 & 11.55 & 23.7 & 5.58 \\
\hline 14 & & $25.3^{*}$ & 22.4 & 11.66 & 20.3 & 19.76 & 24.7 & 2.37 & 26.9 & 6.32 \\
\hline 15 & & 4.4 & 7.3 & 65.68 & 4.1 & 6.82 & 7.5 & 70.45 & 4.4 & 0.00 \\
\hline 16 & & 3.4 & 6.2 & 82.65 & 2.8 & 17.65 & 6.3 & 85.29 & 3.0 & 11.76 \\
\hline SEC & & & 2.69 & & 3.20 & & 3.04 & & 1.72 & \\
\hline SEP & & & 2.28 & & 2.53 & & 1.92 & & 0.83 & \\
\hline $\mathrm{R}^{2}$ & & & 0.915 & & 0.989 & & 0.888 & & 0.992 & \\
\hline
\end{tabular}
hyperspectral images in FFBs was best for weight estimation. However, larger samples size will be necessary to further improve the estimation accuracy.

Table 2 Weight determination by using color and hyperspectral images

* is noted for big size FFB. 
Table 3 Validation results of FFBs ripeness determination by using Euclidean distance in RGB color images

\begin{tabular}{|c|c|c|c|c|c|c|c|c|}
\hline \multirow{2}{*}{ Ripeness } & \multicolumn{4}{|c|}{ Automatic sampling method } & \multicolumn{4}{|c|}{ Manual sampling method } \\
\hline & Overripe & Ripe & Underripe & Unripe & Overripe & Ripe & Underripe & Unripe \\
\hline Overripe & 0 & 50 & 50 & 0 & 100 & 0 & 0 & 0 \\
\hline Ripe & 50 & 0 & 50 & 0 & 0 & 50 & 50 & 0 \\
\hline Underripe & 0 & 0 & 100 & 0 & 0 & 50 & 50 & 0 \\
\hline Unripe & 0 & 0 & 0 & 100 & 0 & 0 & 0 & 100 \\
\hline
\end{tabular}

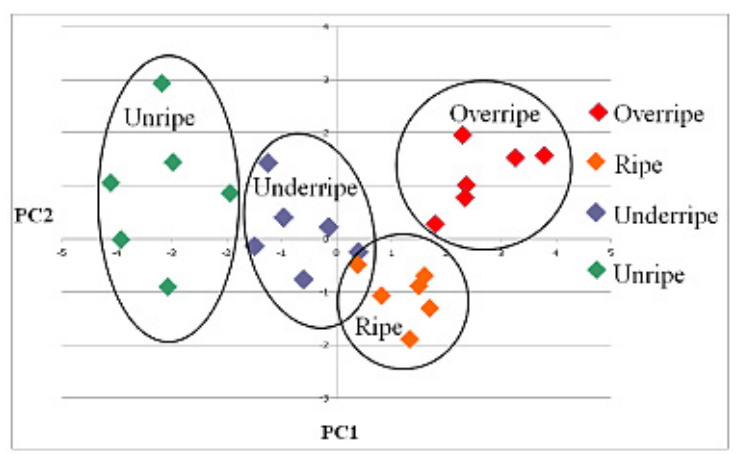

(a) Manual sampling method

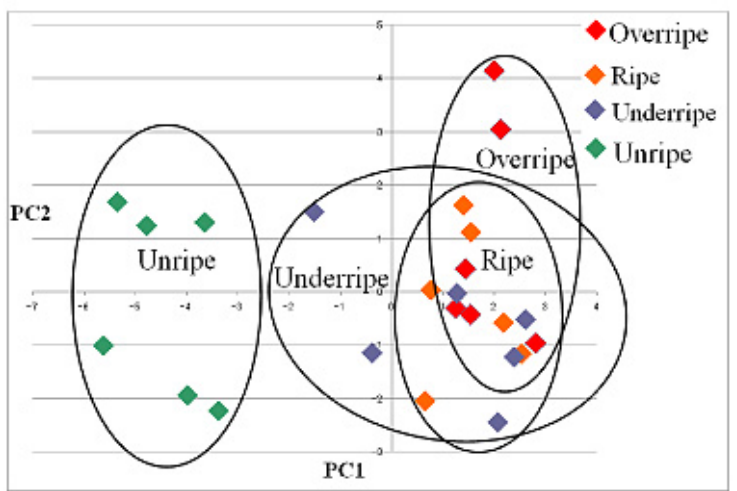

(b) Automatic sampling method

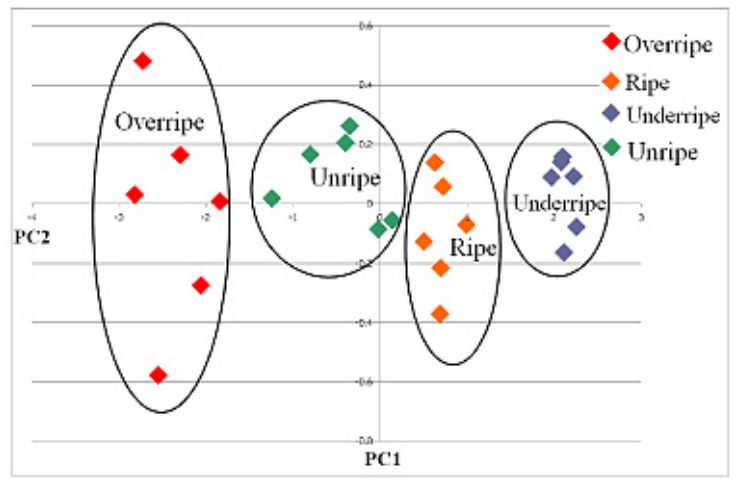

(c) Manual sampling method

Fig. 7 Principle components analysis (PCA) of pixels in FFBs: (a), (b) RGB color image and (c) hyperspectral images

\section{Ripeness estimation}

\section{Ripeness estimation by RGB color images}

The results of the ripeness estimation from RGB color images are shown in Table 3. The results of the principle components analysis (PCA) show that the distribution of the component 1
(PC1) and the component 2 (PC2) of manual sampling method can classify overripe and unripe bunches from other classes of ripeness as shown in Fig. 7(a). The success rate of the ripeness estimation by the manual sampling method indicates that Euclidean distance can classify overripe and unripe bunches from samples. The results obtained by the automatic sampling method are shown in Fig. 7(b). Unripe bunches can be classified from other classes, but classification of overripe, ripe, and underripe bunches is more difficult. This difficulty may be caused in part by the fact that color data from the bunch's spikelet were included in the automatic selection.

Therefore, further research will be needed for more accurate classification using RGB data from overripe, ripe and underripe bunches. Ripeness estimation by the manual sampling method may have more potential application than the automatic sampling method.

\section{Ripeness classification by hyperspectral images}

Fig. 8 shows average intensity spectra in the range of 400$1000 \mathrm{~nm}$ of FFBs collected at four classes of ripeness. The results of the distribution of component 1 (PC1) and the component 2 (PC2) obtained by principle component analysis indicated good performance of classification for all classes of ripeness, as shown in Fig. 7(c). Centroids representing classes of ripeness can be easy to define. The ripeness estimation results using the Euclidean distances gave a good classification rate, as shown in Table 4. When compared with RGB color images, hyperspectral images showed a greater potential for ripeness estimation in oil palm bunches.

\section{Conclusions}

The aim of the present study was to estimate the weight and ripeness of oil palm bunches in order to find a potential application for an in-line monitoring system. RGB color and hyperspectral images were employed.

For weight estimation, the results showed potential application for estimating the weight of FFBs at the same level in both kinds of images. The accuracy rates of weight estimation were $98.9 \%$ and $99.2 \%$ in RGB color and hyperspectral images, respectively. An application of RGB color images may have some advantages for low-cost weight estimation.

For ripeness estimation, differences in color data of concealed 


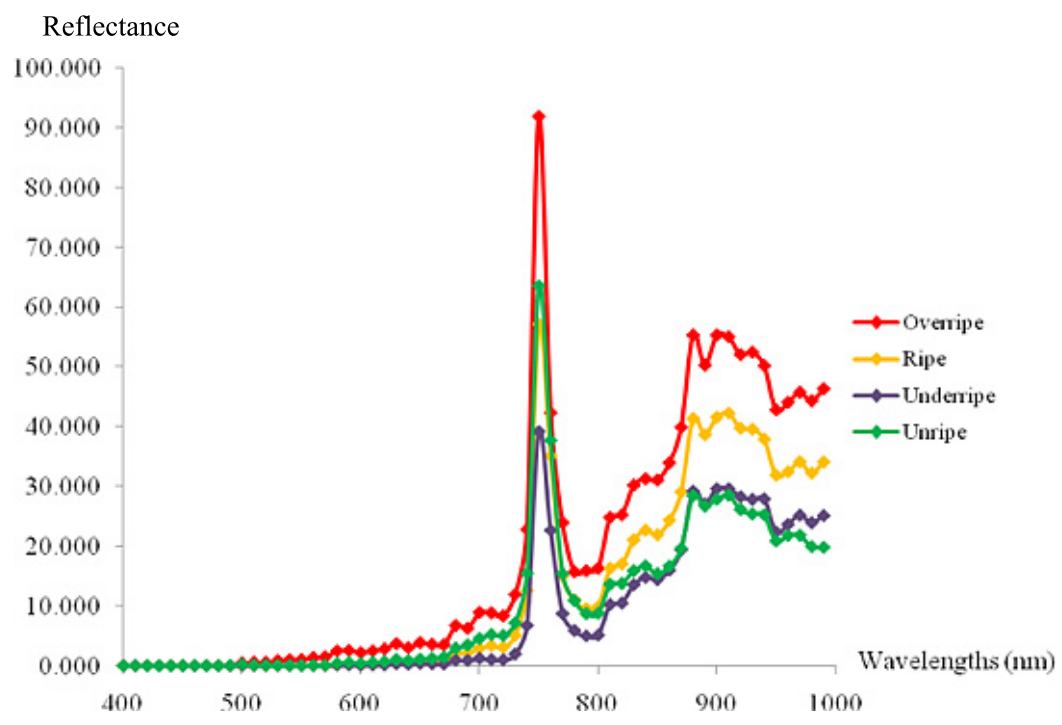

Fig. 8 A typical reflectance of oil palm bunch in different classes of ripeness

Table 4 Validation results of FFB ripeness determination by using Euclidean distance in hyperspectral images

\begin{tabular}{lcrcc}
\hline \multirow{2}{*}{ Ripeness } & \multicolumn{4}{c}{ Manual sampling method } \\
\cline { 2 - 5 } & Overripe & Ripe & Underripe & Unripe \\
\hline Overripe & 100 & 0 & 0 & 0 \\
Ripe & 0 & 100 & 0 & 0 \\
Underripe & 0 & 0 & 100 & 0 \\
Unripe & 0 & 0 & 0 & 100 \\
\hline
\end{tabular}

and not-concealed fruits were employed. Pixels of fruits were obtained by a manual or automatic sampling method.

For color images, a manual sampling method allowed for good classification in overripe and unripe bunches, but misclassification occurred between underripe and ripe bunches. This misclassification may be acceptable for sorting unripe and overripe bunches from purchased bunches in the factory. Conversely, misclassification in overripe, ripe, and underripe bunches occurred with the automatic sampling method. This method is therefore impractical for sorting oil palm bunches. More image processing and classification techniques are needed to improve the accuracy of the automatic sampling method.

For hyperspectral images, concealed fruits were utilized for ripeness estimation by a manual sampling method. Correct classification results in all classes of ripeness were gained, which may indicate that data from hyperspectral images can provide more suitable application of ripeness determination than color images.

\section{References}

Abdullah, M. Z., L. C. Guan, A. M. D. Mohamed and M. A. M. Noor (2002) Color vision system for ripeness inspection of oil palm Elaeis guineensis, Journal of Food Processing Preservation , 26: 213-235.
Abdullah, M. Z., L. C. Guanand and B. M. N. Mohd Azemi (2001) Stepwise discriminant analysis for color grading of oil palm using machine vision system, Transaction of Institute of Chemical Engineering: Part C, 79: 223-231.

Abdullah, M. Z., L. C. Guan, K. C. Limand and A. A. Karim (2004) The applications of computer vision system and tomographic radar imaging for assessing physical properties of food, Journal of Food Engineering, 61: 125-135.

Al-Mallahi, A., T. Kataoka and H. Okamoto (2008) Discrimination between potato tubers and clods by detecting the significant wavebands, Biosystem Engineering, 100: 329-337.

Demirbas, A. (2007) Importance of biodiesel as transportation fuel, Energy Policy, 35 :4661-4670.

Du, C. and D. Sun (2004) Recent developments in the applications of image processing techniques for food quality evaluation, Trends in Food Science \& Technology, 15: 230-249.

Food and Agriculture Organization of the United Nations (FAO) (2007) FAOSTAT, < http://faostat.fao.org >, browed on May 14, 2008.

Gamal, E., W. Ning, E. Adel and N. Michael (2007) Hyperspectral imaging for nondestructive determination of some quality attributes for strawberry, Journal of Food Engineering, 81: 98-107.

Gowen, A. A., C. P. O’Donnell, P. J. Cullen, G. Donwey and J. M. Frias (2007) Hyperspectral imaging-an emerging process analytical tool for food quality and safety control, Trend in Food Science \& Technology, 18: 590-598.

Ismail, W. I. Z., M. Z. Bardie and A. M. A. Hamid (2000) Optical properties for mechanical harvester of oil palm FFB, Journal of Oil Palm Research, 12: 38-45.

Kalam, M. A. and H. H. Masjuki (2002) Biodiesel from palm oil- an analysis of its properties and potential, Biomass and Bioenergy, 23: 471-479.

Leelawath, W. (2006) Expanding planting areas: is it the one and only way to boost up oil palm production?, International Institute for Trade and Development, Bangkok, Thailand, < http:/www.itd.or.th/ th/node/275>, browsed on Aug. 15, 2007.

Leng, T. (2002) Mechanisation in oil palm plantations: achievement and challenges, Malaysian Oil Science and Technology, 22: 70-77.

Murphy, J. D. (2007) Future prospects for oil palm in the $21^{\text {st }}$ century: Biological and related challenges, European Journal of Lipid Sci- 
ence and Technology, 109: 296-306.

Okamoto, H., K. Sakai, T. Murata, T. Kataoka and S. Hata (2006) Object-oriented software framework developed for agricultural hyperspectral imaging analysis, Agricultural Information Research, 15: 103-112. (in Japanese)

Okamoto, H., T. Murata, T. Kataoka and S. Hata (2007) Plant classification for weed detection using hyperspectral imaging with wavelet analysis, Weed Biology and Management, 7: 31-37.

Park, B., W. R. Windham, K. C. Lawrenc and D. P. Smith (2007) Contaminant classification of poultry hyperspectral imagery using a spectral angle mapper algorithm, Biosystem Engineering, 96: 323333.

Patrick, M. M., C. Yud-Ren, S. K. Moon and E. C. Diane (2004) Development of hyperspectral image technique for the detection of apple surface defects and contaminations, Journal of Food Engineering, 61: 67-81.

Sarin, R., M. Sharma, S. Sinharay and R. K. Malhotra (2007) Jatropha-
Palm biodiesel blends: An optimum mix for Asia, Fuel, 86: 13651371 .

Thomas, R. L., P. Sew, C. K. Mok, K. W. Chan, P. T. Easau and S. C. Ng (1971) Fruit ripening in the oil-palm Elaeis guineensis, Annals of Botany, 35: 1219-1925.

Usta, N., E. Öztürk, Ö. Can, E. S. Conkur, S. Nas, A. H. Çon, A. C. Can, M. Topcu (2005) Combustion of biodiesel fuel produced from hazelnut soapstock/waste sunflower oil mixture in Diesel engine. Energy conversion and management, 46:741-755.

Vizhanyo, T. and J. Felfodi (2000) Enhancing colour differences in images of diseased mushrooms, Computers and Electronics in Agriculture, 26: 187-198.

Received October 1, 2008 Accepted January 7, 2009 Information engineering 